Submitted to Physical Review B

FSU-SCRI-96-1214

cond-mat/xxxxxx

\title{
A Plaquette Basis for the Study of Heisenberg Ladders
}

\author{
J. Piekarewicz \\ Supercomputer Computations Research Institute, \\ Florida State University, Tallahassee, FL 32306 \\ J.R. Shepard \\ Department of Physics, \\ University of Colorado, Boulder, CO 80309
}

(August 22, 2018)

\begin{abstract}
We employ a plaquette basis - generated by coupling the four spins in a $2 \times 2$ lattice to a well-defined total angular momentum - for the study of Heisenberg ladders with antiferromagnetic coupling. Matrix elements of the Hamiltonian in this basis are evaluated using standard techniques in angular-momentum (Racah) algebra. We show by exact diagonalization of small $(2 \times 4$ and $2 \times 6)$ systems that in excess of $90 \%$ of the ground-state probability is contained in a very small number of basis states. These few basis states can be used to define a severely truncated basis which we use to approximate low-lying exact eigenstates. We show how, in this low-energy basis, the isotropic spin-1/2 Heisenberg ladder can be mapped onto an anisotropic spin-1 ladder for which the coupling along the rungs is much stronger than the coupling between the rungs. The mapping thereby generates two distinct energy scales which greatly facilitates understanding the dynamics of the original spin- $1 / 2$ ladder. Moreover, we use these insights to define an effective low-energy Hamiltonian in accordance to the newly developed COntractor REnormalization group (CORE) method. We show how a simple range-2 CORE approximation to the effective Hamiltonian to be used with our truncated basis reproduces the low-energy spectrum of the exact $2 \times 6$ theory at the $\lesssim 1 \%$ level.
\end{abstract}

\section{INTRODUCTION}

Interest in the study of ladder compounds has been stimulated by suggestions that these deceptively simple materials could exhibit some of the critical behavior believed to be responsible for high-temperature (high- $T_{c}$ ) superconductivity [1,22]. Moreover, these ladder materials - built one chain at a time - could bridge the transition from the one-dimensional 
(1D) chains, where large simulations are carried out routinely, to the two-dimensional (2D) structures that are known to be at the heart of the high- $T_{c}$ compounds [3]. Although this might very well be the case, the road from one to two dimensions has been full of (quantum) surprises [2]. Indeed, it is now known that some of the properties common to the 1D- and 2D-systems - such as the absence of a spin gap - are only shared by the odd-leg ladders. In contrast, even-leg ladders have a finite spin gap which should manifest itself in the form of an exponential behavior of the magnetic susceptibility at low temperatures; this (exponential) activation has been confirmed experimentally [ब. Finally, the excitement about the ladder materials has recently been fueled even further by some preliminary reports that suggest a superconducting transition in some physical realizations of the ladders at a critical temperature of about $T_{c}=12 \mathrm{~K}$ [5].

An arsenal of numerical approaches has been employed to elucidate the physics of the ladder materials. This includes exact and Lanczos diagonalization techniques [6], quantum Monte-Carlo methods [7], and a density-matrix-renormalization-group approach [8]. While these techniques have achieved a high degree of sophistication, the selection of a basis has not. Indeed, the overwhelming majority - if not all - of the calculations reported in the literature have rely on the traditional " $S_{z}$ " basis. Undoubtedly, the biggest advantage of the $S_{z}$ basis is its simplicity. Indeed, matrix elements of the Hamiltonian are trivially computed in this basis. Yet, it is unlikely that the $S_{z}$ basis represents the "optimal" choice for the study of Heisenberg antiferromagnets with isotropic coupling.

Recent discussions of the possible utility of alternative bases for the study of the ladder materials by Martins, Dagotto, and Riera [9] have stimulated us to examine this issue. The initial step in our program was to rediscover the "plaquette" basis [10] and then to examine how it might be used in the study of spin ladders. A particularly appealing feature of the plaquette basis - generated from the coupling of the four spins in a $2 \times 2$ lattice to a well-defined total angular momentum - is that the states in the basis are the eigenstates of the largest two-leg ladder that can be solved in closed form. By this mere fact, it is clear that much of the important physics of the problem has been incorporated into the basis. Obviously, an important component of our program is the evaluation of matrix elements of the Hamiltonian. It is for this that we rely heavily on the sophisticated apparatus of angularmomentum algebra [11, 12] that has been developed in atomic [13] and nuclear physics [14, 15] over many years.

Our paper has been organized as follows. In Sec. [1] we describe the plaquette basis and compute all the necessary matrix elements of the Hamiltonian. In Sec. IIT we study small $2 \times 4$ and $2 \times 6$ ladders to illustrate the advantage of our approach. In particular, we define a severely truncated basis which includes only the 4 lowest, out of 16, 1-plaquette energy levels. We use the newly developed COntractor REnormalization (CORE) 16 method to construct a low-energy effective Hamiltonian to be used with the truncated basis and find that the exact eigenvalues of the 3-plaquette system are very accurately reproduced. Finally, we summarize in Sec. 【V.

\section{FORMALISM}

The focus of our paper is the plaquette basis. This basis is generated from the coupling of the four spins in a $2 \times 2$ plaquette to a well-defined total angular momentum. To define the 
basis and to illustrate most of the techniques it is sufficient to concentrate on the $2 \times 4$ (or two-plaquette) antiferromagnet with isotropic nearest-neighbor coupling $J \equiv 1$. Moreover, we restrict ourselves to the $S=1 / 2$ case, although the formalism can be applied with few modifications to arbitrary spin. Indeed, we exploit this flexibility when, in Sec [II], we discuss spin-1 ladders which are in some sense equivalent to the spin-1/2 ladders discussed in this section. The model Hamiltonian is given - adopting open boundary conditions - by

$$
H=\sum_{\langle i, j\rangle} \mathbf{S}_{i} \cdot \mathbf{S}_{j} \equiv H_{0}(1)+H_{0}(2)+V(1,2)
$$

where $\langle i, j\rangle$ denotes nearest-neighbor sites and

$$
\begin{aligned}
H_{0}(1) & \equiv \mathbf{S}_{1} \cdot \mathbf{S}_{2}+\mathbf{S}_{1} \cdot \mathbf{S}_{3}+\mathbf{S}_{2} \cdot \mathbf{S}_{4}+\mathbf{S}_{3} \cdot \mathbf{S}_{4}, \\
H_{0}(2) & \equiv \mathbf{S}_{5} \cdot \mathbf{S}_{6}+\mathbf{S}_{5} \cdot \mathbf{S}_{7}+\mathbf{S}_{6} \cdot \mathbf{S}_{8}+\mathbf{S}_{7} \cdot \mathbf{S}_{8}, \\
V(1,2) & \equiv \mathbf{S}_{3} \cdot \mathbf{S}_{5}+\mathbf{S}_{4} \cdot \mathbf{S}_{6} .
\end{aligned}
$$

Note that this simple two-leg ladder is formed from two interacting $n=4$ spin chains; spins on the left(right) chain are labeled with odd(even) numbers. Moreover, $H_{0}$ represents the Hamiltonian of an isolated $2 \times 2$ system, while $V(1,2)$ is the coupling "potential". The $2 \times 2$ Hamiltonian $H_{0}$ - a simple "antiferromagnet" [10 - can be re-written in the following form:

$$
H_{0}(1)=\left(\mathbf{S}_{1}+\mathbf{S}_{4}\right) \cdot\left(\mathbf{S}_{2}+\mathbf{S}_{3}\right)=\mathbf{L}_{1} \cdot \mathbf{L}_{2}=\frac{1}{2}\left(\mathbf{J}_{1}^{2}-\mathbf{L}_{1}^{2}-\mathbf{L}_{2}^{2}\right)
$$

where we have defined link and plaquette angular-momentum variables, respectively, as

$$
\mathbf{L}_{1} \equiv \mathbf{S}_{1}+\mathbf{S}_{4} ; \quad \mathbf{L}_{2} \equiv \mathbf{S}_{2}+\mathbf{S}_{3} ; \quad \mathbf{J}_{1} \equiv \mathbf{L}_{1}+\mathbf{L}_{2}=\mathbf{S}_{1}+\mathbf{S}_{2}+\mathbf{S}_{3}+\mathbf{S}_{4}
$$

The physically appealing feature of the plaquette basis is that $H_{0}$ is diagonal in this basis. That is,

$$
H_{0}(1)\left|l_{1} l_{2}, j_{1} m_{1}\right\rangle=\frac{1}{2}\left[j_{1}\left(j_{1}+1\right)-l_{1}\left(l_{1}+1\right)-l_{2}\left(l_{2}+1\right)\right]\left|l_{1} l_{2}, j_{1} m_{1}\right\rangle .
$$

It is interesting to note that one needs to couple the spins along the diagonal - which are the only spins that do not interact - in order to bring the Hamiltonian into a diagonal form.

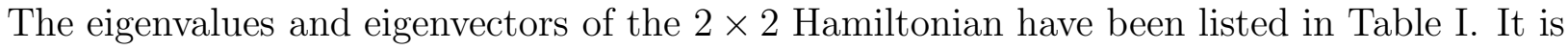
also instructive to write the ground-state of $H_{0}$, namely, the $\left|l_{1}=l_{2}=1, j_{1}=m_{1}=0\right\rangle$ state, in terms of the $S_{z}$ basis. That is,

$$
\left|\Psi_{0}\right\rangle=\frac{1}{\sqrt{3}}\left[|\uparrow \downarrow \downarrow \uparrow\rangle+|\downarrow \uparrow \uparrow \downarrow\rangle-\frac{1}{2}|\uparrow \uparrow \downarrow \downarrow\rangle-\frac{1}{2}|\uparrow \downarrow \uparrow \downarrow\rangle-\frac{1}{2}|\downarrow \uparrow \downarrow \uparrow\rangle-\frac{1}{2}|\downarrow \downarrow \uparrow \uparrow\rangle\right] .
$$

This simple finding, namely the strong fragmentation of the ground-state probability in the $S_{z}$ basis - but not in the plaquette basis - represents one of our central results.

The most challenging part of the calculation in the plaquette basis is the computation of the matrix elements of the coupling potential $V(1,2)$; recall that in the $S_{z}$ basis matrix elements of the Hamiltonian can be evaluated by inspection. Although by no means trivial, the sort of computations which arise in this new basis are done routinely in atomic [13] and 
nuclear physics [14,15]. Indeed, over the years a sophisticated formalism-known generically as Racah algebra - has been developed to tackle these computations [11,12]. It is on these techniques that we rely heavily to compute the matrix elements of $V(1,2)$. We find

$$
\begin{aligned}
& \left\langle\left(l_{1}^{\prime} l_{2}^{\prime}\right) j_{1}^{\prime},\left(l_{3}^{\prime} l_{4}^{\prime}\right) j_{2}^{\prime}, j m|V(1,2)|\left(l_{1} l_{2}\right) j_{1},\left(l_{3} l_{4}\right) j_{2}, j m\right\rangle=\frac{3}{4}(-1)^{l_{2}+l_{3}+j} \widehat{\jmath_{1}} \widehat{\jmath_{2}} \widehat{\jmath_{1}^{\prime}} \widehat{\jmath_{2}^{\prime}}\left\{\begin{array}{lll}
j_{1} & j_{1}^{\prime} & 1 \\
j_{2}^{\prime} & j_{2} & j
\end{array}\right\} \\
& {\left[\delta_{l_{2} l_{2}^{\prime}} \delta_{l_{3} l_{3}} \widehat{l_{1}} \widehat{l_{4}} \widehat{l_{1}} \widehat{l_{4}^{\prime}}\left\{\begin{array}{lll}
l_{1} & l_{1}^{\prime} & 1 \\
j_{1}^{\prime} & j_{1} & l_{2}
\end{array}\right\}\left\{\begin{array}{ccc}
l_{1} & l_{1}^{\prime} & 1 \\
1 / 2 & 1 / 2 & 1 / 2
\end{array}\right\}\left\{\begin{array}{ccc}
l_{4} & l_{4}^{\prime} & 1 \\
j_{2}^{\prime} & j_{2} & l_{3}
\end{array}\right\}\left\{\begin{array}{ccc}
l_{4} & l_{4}^{\prime} & 1 \\
1 / 2 & 1 / 2 & 1 / 2
\end{array}\right\} \pm\right.} \\
& \left.\delta_{l_{1} l_{1}^{\prime}} \delta_{l_{4} l_{4}^{\prime}} \widehat{l_{2}} \widehat{l_{3}} \widehat{l_{2}^{\prime}} \widehat{l}_{3}^{\prime}\left\{\begin{array}{lll}
l_{2} & l_{2}^{\prime} & 1 \\
j_{1}^{\prime} & j_{1} & l_{1}
\end{array}\right\}\left\{\begin{array}{ccc}
l_{2} & l_{2}^{\prime} & 1 \\
1 / 2 & 1 / 2 & 1 / 2
\end{array}\right\}\left\{\begin{array}{ccc}
l_{3} & l_{3}^{\prime} & 1 \\
j_{2}^{\prime} & j_{2} & l_{4}
\end{array}\right\}\left\{\begin{array}{ccc}
l_{3} & l_{3}^{\prime} & 1 \\
1 / 2 & 1 / 2 & 1 / 2
\end{array}\right\}\right] .
\end{aligned}
$$

Note that we have defined $\hat{x} \equiv \sqrt{2 x+1}$ and that the $+/-$ sign in the above expression should be adopted whenever $l_{1}+l_{2}^{\prime}+l_{3}^{\prime}+l_{4}+j_{1}+j_{1}^{\prime}+j_{2}+j_{2}^{\prime}=$ even/odd. Aside from simple phases and numerical factors, the matrix elements of $V(1,2)$ depend on scalar functions (i.e., independent of $m$ ) known as Racah coefficients; here we cast the matrix elements in terms of the more symmetric $6-j$ symbols [11,12]. Closed-form expressions are readily available for the numerical computation of these (and many other) recoupling coefficients [17].

\section{APPLICATIONS}

In the present section we concentrate on small $(2 \times 4$ and $2 \times 6)$ ladders to illustrate some of the advantages of the new basis. Our aim is to provide convincing evidence, by means of a few simple examples, of the utility of the plaquette basis. Hopefully, the evidence will be strong enough to persuade some researchers in the field to exploit this basis in future calculations.

\section{A. Distribution of ground-state strength}

In Fig. 1 we display the ground-state probabilities $\left(\left|\left\langle\alpha \mid \Psi_{0}\right\rangle\right|^{2}\right.$ with $\alpha$ an element of the basis) for the $2 \times 4$ Hamiltonian. This picture emphasizes what was already evident from Eq. (6), namely, a strong fragmentation of ground-state strength among the many (70) states

in the $S_{z}$ basis. Indeed, the only basis states containing a nontrivial amount $(\sim 15 \%)$ of strength are the (staggered) Néel states. In contrast, in the plaquette basis a single state carries $85 \%$ of the ground-state probability and with two states one can practically account for the full probability. Moreover, this picture deteriorates little as one increases the size of the system. Indeed, for a $2 \times 6$ system one basis state - out of 132 - carries in excess of $70 \%$ of the ground-state probability and with only three states one can account for almost $90 \%$ of it. In Fig. 2 we show the corresponding distribution of strength for the first excited state of the system. Most of the features observed for the ground state remain valid in this case as well. Based on this evidence, we believe that the plaquette basis could prove very useful in numerical computations of ladder compounds. 


\section{B. Contractor renormalization group method}

Given that most of the physics of the ground state (and of the low-lying excited states) is contained in a very few numbers of plaquette-basis states, it seems natural to attempt some form of truncation of the basis so that larger systems may be more readily simulated in the computer. The choice becomes obvious upon glancing at Table @; one should retain the first four states, having energies $E=-2$ and $E=-1$ (three-fold degenerate), respectively. Note that with this choice the link angular momenta - the high-energy degrees of freedom in the theory-have been "frozen" at $l_{1}=l_{2}=1$, while the plaquette angular momentum, having values of $j_{1}=0,1$, becomes the effective low-energy degree of freedom in the new theory. In this way the original theory defined on a 16-dimensional Hilbert space (per plaquette) will get mapped into a new effective theory having the same low-energy physics as the original theory, but defined on a Hilbert space of one fourth the size. The low-energy effective theory will now be constructed using the newly developed COntractor REnormalization group (CORE) method [16].

CORE provides a systematic approach at constructing the new low-energy theory using contraction and cluster-expansion techniques. The first step into the implementation of CORE is the selection of an "elementary" block and a truncation scheme. In our case the block is the $2 \times 2$ plaquette and the truncation scheme has been described above; the link variables become frozen and the plaquette variable is limited to take the values $j_{1}=0,1$. Constructing an effective Hamiltonian with the same low-energy properties as the original theory - on a system that contains only one single block $\left(B_{1}\right)$ - is straightforward. We obtain,

$$
\left\langle j_{1}^{\prime} m_{1}^{\prime}\left|H_{\mathrm{eff}}\left(B_{1}\right)\right| j_{1} m_{1}\right\rangle=\left[\frac{1}{2} j_{1}\left(j_{1}+1\right)-2\right] \delta_{j_{1} j_{1}^{\prime}} \delta_{m_{1} m_{1}^{\prime}}
$$

This low-energy Hamiltonian constitutes the range-1 term in the cluster expansion and is denoted by $h_{1}\left(B_{1}\right)=H_{\text {eff }}\left(B_{1}\right)$. One now proceeds to calculate the range- 2 contribution to the cluster expansion by computing an effective Hamiltonian on a system that contains two connected blocks $\left(B_{1}\right.$ and $\left.B_{2}\right)$. This is the $2 \times 4$ Hamiltonian of Eq. (2). In constructing the various contributions to the cluster expansion one must pay particular attention to the overlaps between the exact eigenstates of the original Hamiltonian and the low-energy basis. Since it is simpler to study these overlaps in Hilbert spaces which reflect the symmetries of the Hamiltonian, we work with a low-energy basis of definite total spin; recall that $[H, \mathbf{J}]=0$, where $\mathbf{J} \equiv \mathbf{S}=\sum_{i} \mathbf{S}_{i}$ is the total spin of the system.

We start with the $j=0$ sector. In this sector, there are 14 eigenstates of the exact $2 \times 4$ Hamiltonian, while there are only two low-energy states in the truncated basis, namely, $\left|\phi_{1}\right\rangle \equiv\left|j_{1}=j_{2}=0, j=m=0\right\rangle$ and $\left|\phi_{2}\right\rangle \equiv\left|j_{1}=j_{2}=1, j=m=0\right\rangle$. These two states have a nonzero overlap with the exact ground state $\left|E_{0}^{(0)}\right\rangle$. Indeed, these are the two states that dominate the ground-state probability in Fig. 1. In particular, this implies that both of these basis states will "contract" onto the same eigenstate of the Hamiltonian, i.e.,

$$
\lim _{t \rightarrow \infty} e^{-t H}\left|\phi_{1}\right\rangle \propto\left|E_{0}^{(0)}\right\rangle \text { and } \lim _{t \rightarrow \infty} e^{-t H}\left|\phi_{2}\right\rangle \propto\left|E_{0}^{(0)}\right\rangle
$$

CORE demands that only one low-energy basis state should contract into the ground state. CORE also offers a simple solution to this problem: construct a new truncated basis by 
performing a similarity transformation on the original one so that each state in the new basis $\left(\xi_{i}\right)$ contracts onto a unique eigenstate of the exact Hamiltonian, i.e.,

$$
\lim _{t \rightarrow \infty} e^{-t H}\left|\xi_{1}\right\rangle \propto\left|E_{0}^{(0)}\right\rangle \text { but } \lim _{t \rightarrow \infty} e^{-t H}\left|\xi_{2}\right\rangle \propto\left|E_{1}^{(0)}\right\rangle
$$

where $E_{1}^{(0)}$ is the second lowest eigenvalue of the Hamiltonian in the $j=0$ sector. This is all that is needed. In this way, the relevant matrix elements of the effective Hamiltonian in the $j=0$ sector become:

$$
\begin{aligned}
& \left\langle j_{1}^{\prime} j_{2}^{\prime} j=0\left\|H_{\text {eff }}\left(B_{1}, B_{2}\right)\right\| j_{1} j_{2} j=0\right\rangle= \\
& \quad\left(\begin{array}{rr}
\cos \theta_{0} & -\sin \theta_{0} \\
\sin \theta_{0} & \cos \theta_{0}
\end{array}\right)\left(\begin{array}{cc}
E_{0}^{(0)} & 0 \\
0 & E_{1}^{(0)}
\end{array}\right)\left(\begin{array}{rr}
\cos \theta_{0} & \sin \theta_{0} \\
-\sin \theta_{0} & \cos \theta_{0}
\end{array}\right),
\end{aligned}
$$

where $E_{0}^{(0)}=-4.293, E_{1}^{(0)}=-2.500$, and $\theta_{0}=-18.482^{\circ}$.

The construction of the effective Hamiltonian in the $j=1$ sector proceeds in a similar fashion. In this sector there are 28 eigenstates and three low-energy basis states (each with a three-fold degeneracy). These are: $\left|\phi_{3}\right\rangle \equiv\left|j_{1}=1 j_{2}=0, j=1 \mathrm{~m}\right\rangle,\left|\phi_{4}\right\rangle \equiv \mid j_{1}=0 j_{2}=1, j=$ $1 \mathrm{~m}\rangle$, and $\left|\phi_{5}\right\rangle \equiv\left|j_{1}=j_{2}=1, j=1 \mathrm{~m}\right\rangle$. Since, in this particular case, only the first two states need to be transformed, the similarity transformation can again be parameterized in terms of a single angle. That is,

$$
\begin{aligned}
& \left\langle j_{1}^{\prime} j_{2}^{\prime} j=1\left\|H_{\text {eff }}\left(B_{1}, B_{2}\right)\right\| j_{1} j_{2} j=1\right\rangle= \\
& \left(\begin{array}{ccc}
\cos \theta_{1} & -\sin \theta_{1} & 0 \\
\sin \theta_{1} & \cos \theta_{1} & 0 \\
0 & 0 & 1
\end{array}\right)\left(\begin{array}{ccc}
E_{0}^{(1)} & 0 & 0 \\
0 & E_{1}^{(1)} & 0 \\
0 & 0 & E_{2}^{(1)}
\end{array}\right)\left(\begin{array}{ccc}
\cos \theta_{1} & \sin \theta_{1} & 0 \\
-\sin \theta_{1} & \cos \theta_{1} & 0 \\
0 & 0 & 1
\end{array}\right),
\end{aligned}
$$

where $E_{0}^{(1)}=-3.523, E_{1}^{(1)}=-2.915, E_{2}^{(1)}=-2.590$, and $\theta_{1}=45^{\circ}$.

Finally, since (up to a five fold degeneracy) there is a unique $j=2$ state in the low energy basis, namely, $\left|\phi_{6}\right\rangle \equiv\left|j_{1}=j_{2}=1, j=2 m\right\rangle$, the effective Hamiltonian in this sector is simply given by

$$
\left\langle j_{1}^{\prime} j_{2}^{\prime} j=2\left\|H_{\mathrm{eff}}\left(B_{1}, B_{2}\right)\right\| j_{1} j_{2} j=2\right\rangle=E_{0}^{(2)}=-2.207 \text {. }
$$

Collecting all the above results we can now write the effective $2 \times 4$ low-energy Hamiltonian in the direct product basis:

$$
\begin{aligned}
& \left\langle j_{1}^{\prime} m_{1}^{\prime}, j_{2}^{\prime} m_{2}^{\prime}\left|H_{\mathrm{eff}}\left(B_{1}, B_{2}\right)\right| j_{1} m_{1}, j_{2} m_{2}\right\rangle=\delta_{m_{1}^{\prime}+m_{2}^{\prime}, m_{1}+m_{2}} \\
& \quad \sum_{j}\left\langle j_{1}^{\prime} j_{2}^{\prime} j \| H_{\mathrm{eff}}\left(B_{1}, B_{2}\right)|| j_{1} j_{2} j\right\rangle\left\langle j_{1}^{\prime} m_{1}^{\prime}, j_{2}^{\prime} m_{2}^{\prime} \mid j m_{1}^{\prime}+m_{2}^{\prime}\right\rangle\left\langle j_{1} m_{1}, j_{2} m_{2} \mid j m_{1}+m_{2}\right\rangle,
\end{aligned}
$$

where $\left\langle j_{1} m_{1}, j_{2} m_{2} \mid j m\right\rangle$ are Clebsch-Gordan coefficients. The range-2 contribution to the cluster expansion is obtained by simply removing from $H_{\text {eff }}\left(B_{1}, B_{2}\right)$ those range-1 terms that have already been included in the single-block calculation, i.e.,

$$
h_{2}\left(B_{1}, B_{2}\right)=H_{\mathrm{eff}}\left(B_{1}, B_{2}\right)-h_{1}\left(B_{1}\right)-h_{1}\left(B_{2}\right) .
$$

To construct the renormalized Hamiltonian one must continue this procedure, indefinitely, on larger and larger connected blocks. Here we will stop at the range-2 contribution. 
Note that for our choice of basis, this range- 2 approximation already takes into account correlations among next-to-next nearest neighbors. The approximate (up to range-2) renormalized Hamiltonian becomes

$$
H_{\mathrm{ren}}=\sum_{j=1}^{\infty}\left[h_{1}\left(B_{j}\right)+h_{2}\left(B_{j}, B_{j+1}\right)\right] .
$$

It is instructive to use this approximation to compute the low-energy spectrum of the $2 \times 6$ (three-plaquette) Heisenberg antiferromagnet. For this case, the range-2 approximation yields

$$
H_{\text {ren }}=h_{1}\left(B_{1}\right)+h_{1}\left(B_{2}\right)+h_{1}\left(B_{3}\right)+h_{2}\left(B_{1}, B_{2}\right)+h_{2}\left(B_{2}, B_{3}\right) .
$$

This expression is useful as it suggests when the cluster expansion might become rapidly convergent. If an optimal basis has been chosen - provided that one exists - one might hope that most of the low-energy spectrum could be generated by the range-1 terms, leaving the range- 2 terms in charge of the fine tuning. Alternatively, an optimal basis could generatedynamically - two energy scales in the problem; a large one associated with physics within the blocks and a small one associated with the "residual interaction" between the blocks. Our investigations show that - for our truncated basis - such is indeed the case. First, one can simply compare the individual matrix elements of $h_{1}$ and $h_{2}$. Those of the former are typically 4 to 8 times larger than those of the latter. Second, one can arrive at the same conclusion by mapping the original isotropic spin- $1 / 2$ ladder onto an equivalent anisotropic spin-1 ladder as follows. Note that for the 2-plaquette system [see Eq. (2)]

$$
H_{0}(1)+H_{0}(2)=\left(\mathbf{S}_{1}+\mathbf{S}_{\mathbf{4}}\right) \cdot\left(\mathbf{S}_{\mathbf{2}}+\mathbf{S}_{\mathbf{3}}\right)+\left(\mathbf{S}_{\mathbf{5}}+\mathbf{S}_{\mathbf{8}}\right) \cdot\left(\mathbf{S}_{\mathbf{6}}+\mathbf{S}_{\mathbf{7}}\right)=\mathbf{L}_{\mathbf{1}} \cdot \mathbf{L}_{\mathbf{2}}+\mathbf{L}_{\mathbf{3}} \cdot \mathbf{L}_{\mathbf{4}} \cdot
$$

In the truncated basis, all link angular momenta are 1 ; hence the $\mathbf{L}_{\mathbf{i}}$ are spin-1 operators. Now consider $V(1,2)=\left(\mathbf{S}_{3} \cdot \mathbf{S}_{5}+\mathbf{S}_{4} \cdot \mathbf{S}_{6}\right)$. In the truncated basis, symmetries permit the interaction to be written as

$$
\begin{aligned}
V(1,2) & =\mathbf{S}_{3} \cdot \mathbf{S}_{5}+\mathbf{S}_{4} \cdot \mathbf{S}_{\mathbf{6}} \\
& \rightarrow \frac{1}{4}\left[\left(\mathbf{S}_{\mathbf{2}}+\mathbf{S}_{\mathbf{3}}\right) \cdot\left(\mathbf{S}_{\mathbf{5}}+\mathbf{S}_{\mathbf{8}}\right)+\left(\mathbf{S}_{\mathbf{1}}+\mathbf{S}_{4}\right) \cdot\left(\mathbf{S}_{\mathbf{6}}+\mathbf{S}_{\mathbf{7}}\right)\right] \\
& =\frac{1}{4}\left[\mathbf{L}_{\mathbf{1}} \cdot \mathbf{L}_{4}+\mathbf{L}_{\mathbf{2}} \cdot \mathbf{L}_{\mathbf{3}}\right] .
\end{aligned}
$$

Hence, in the truncated basis - which carries much of the important physics of the problemthe isotropic 2-plaquette spin-1/2 system is equivalent to a 1-plaquette spin-1 system where the coupling along the rungs $\left(\mathbf{L}_{1} \cdot \mathbf{L}_{2}+\mathbf{L}_{3} \cdot \mathbf{L}_{4}\right)$ is four times as strong as the coupling between the rungs $\left(\frac{1}{4}\left[\mathbf{L}_{1} \cdot \mathbf{L}_{4}+\mathbf{L}_{\mathbf{2}} \cdot \mathbf{L}_{\mathbf{3}}\right]\right)$. In this context our basis is optimal in the sense that the much stronger "rung" couplings are diagonal in it. We also note that this mapping, in conjunction with CORE techniques similar to those discussed above, is likely to provide a useful starting point for formulating a renormalization group transformation which can permit us to estimate properties of infinite ladders. This will be the topic of a future publication.

In Table II we display the low-energy spectrum of the $2 \times 6$ Hamiltonian using a variety of approximations; the ratio to the exact value appears in parenthesis. Recall that the 
spectrum has been computed with open boundary conditions. The states have been classified according to their total spin, which is listed in the first column. In the second column we report the results from an extreme "weak-coupling" calculation. In this approximation the $2 \times 2$ plaquettes are treated exactly but the residual interaction between the plaquettes is neglected [i.e., $V(j, j+1) \equiv 0$; see Eq. (2)]. We observe that at the 80 to 90 percent level, the spectrum is, indeed, accounted for by the mere selection of the basis. In the third column we report a calculation which uses the exact $2 \times 6$ Hamiltonian but with the truncated low-energy basis. Departures from the exact results are only 5 to $10 \%$. In principle, this truncation could enable the simulations of larger systems, as the number of states increases with the number of plaquettes $\left(N_{p}\right)$ only as $4^{N_{p}}$, rather than as $4^{2 N_{p}}$. However, in many applications, this level of accuracy may still be insufficient. Amazingly, a dramatic improvement on these calculations results from expending the very little additional effort required to construct the CORE effective Hamiltonian for the truncated basis. As shown in Table II. COREimproved calculations yield results that range from a fraction of $1 \%$ to a few percent of the exact answer. Note that these results were obtained by the diagonalization of - at most$20 \times 20$ matrices; instead, the exact calculation in the $S_{z}$ basis requires a diagonalization of a $924 \times 924$ matrix. In the near future, we plan to use this renormalized Hamiltonian, perhaps including range-3 contributions, to simulate larger systems.

We conclude this section with a brief comment about the doping of the ladders. In order to gain some qualitative insight into the nature of hole correlations, Dagotto and collaborators introduced two energy scales in the problem: a large exchange coupling $J^{\prime}$ along the rungs relative to a small exchange coupling $J$ along the chains [四]. They observed that if a pair of holes is added to the system, the energy will get minimized whenever the two holes go into the same rung in the ladder. Further, they concluded by means of numerical evidence, that most of the arguments developed for the anisotropic case remain valid even when $J^{\prime} \approx J$; note that to date, the physical realization of the ladders seem to obey the isotropic relation [Q] $]$. In this paper we have only considered the isotropic case. A particularly gratifying aspect of the mapping described above is the natural appearance in the isotropic system of distinct energy scales in the effective spin-1 ladder. This appears to permit carrying over the arguments of Dagotto and collaborators with little modification. Now it is the rungs on the spin-1 ladder which are strongly bound, consisting of a pair of "frozen" spin triplets coupled to an overall angular momentum of zero; interactions between rungs are relatively weak. In terms of the original spin- $1 / 2$ ladder, we may conclude that individual plaquettes interact only weakly. Hence the weak-coupling limit defined above should be a reasonable approximation when applied to very large systems just as we have found it to be by comparing with exact results for the 3-plaquette case. In this limit it is simple to see that the ground-state of the system consists of all plaquettes being in the lowest $j=0$ state, with energy per plaquette of -2 (see Table [). When a pair of holes is introduced into the system the holes can go into two different plaquettes at a cost in energy of +2 (the lowest energy of three spins in a plaquette is equal to -1 ). Alternatively, the holes can go into a rung - or along a chain - in the same plaquette at a cost in energy of $+5 / 4$ (the lowest energy of two spins in a plaquette is equal to $-3 / 4$ ). Hence, it becomes energetically favorable for the two holes to bind and break as few $j=0$ plaquettes as possible. Moreover, as another pair of holes is added into the system, it becomes energetically favorable - at least for holes with no mobility - for the four holes to go into the same plaquette, rather 
than for the new pair to break another plaquette.

\section{CONCLUSIONS}

We have employed a plaquette basis for the study of antiferromagnetic Heisenberg ladders. The states in the basis represent the eigenstates of the $2 \times 2$ plaquette and are constructed from the angular-momentum coupling of the four spins in the plaquette. Matrix elements of the Hamiltonian were computed in this basis and were expressed in terms of a product of five Racah coefficients. These expressions are considerably more complicated than the corresponding ones obtained using the conventional $S_{z}$ basis. Yet, they can be efficiently computed by employing angular-momentum techniques that have been developed over the years in atomic and nuclear physics. Moreover, this basis seems to capture some of the important physics of these complicated systems. Indeed, we have shown that the distribution of ground-state and first-excited-state strength is concentrated in a very few number of states. This is in contrast to the $S_{z}$ basis where the strength is strongly fragmented. This concentration of strength among a few states provides a very natural truncation scheme for the basis. We selected a low-energy basis which reduces the size of the Hilbert space by a factor of four per plaquette. By using the original $(2 \times 6)$ Hamiltonian in this truncated space we obtained a low-energy spectrum that was within $10 \%$ of the exact answer. However, by improving the Hamiltonian - via CORE - we were able to get within $1 \%$ of the exact answer. Moreover, the mere selection of the basis dynamically generates two-energy scales in the problem - even in the case of an isotropic coupling. This phenomenon is most clearly understood by mapping the original isotropic spin-1/2 ladder onto an effective spin-1 ladder in which the coupling along rungs is four times stronger than the coupling between

rungs. This separation of scales is important for the development of qualitative insights into the nature of hole doping. In particular, it supports the notion that holes will go into the ladders in such a way as to disturb the minimum number of $j=0$ plaquettes.

In summary, we have provided solid evidence in support of a plaquette basis for the study of Heisenberg ladders. Much work remains to be done, such as the implementation of a Lanczos diagonalization procedure and the study of larger systems using the renormalized range-2 (or even range-3) CORE Hamiltonian. Yet, we believe that the mere selection of a basis could play a prominent role in the elucidation of the important physics behind the ladder materials.

\section{ACKNOWLEDGMENTS}

We thank E. Dagotto for introducing one of us (JP) to some of the issues addressed in this work and for many useful and stimulating discussions. This work was supported by the DOE under Contracts Nos. DE-FC05-85ER250000, DE-FG05-92ER40750 and DE-FG0393ER40774. 


\section{REFERENCES}

[1] E. Dagotto, J. Riera, and D.J. Scalapino, Phys. Rev. B 45, 5744 (1992).

[2] E. Dagotto and T.M. Rice, Science 271, 618 (1996).

[3] E. Dagotto, Rev. Mod. Phys. 66, 763 (1994).

[4] M. Azuma, Z. Hiroi, M. Takano, K. Ishida, and Y. Kitaoka, Phys. Rev. Lett. 73, 3463 (1994).

[5] M. Uehara, T. Nagata, J. Akimitsu, H. Takahashi, N. Mori, and K. Kinoshita, J. Phys. Soc. Japan, in press.

[6] C. Lanczos, J. Res. Nat. Bur. Stand. 45, 255 (1950).

[7] K. Binder and D.W. Heermann, Monte Carlo Simulations in Statistical Physics, Springer-Verlag (Berlin/Heidelberg/New York) 1992.

[8] S.R. White, Phys. Rev. Lett. 69, 2863 (1992).

[9] G.B. Martins, E. Dagotto, and J. Riera, in preparation.

[10] N.W. Ashcroft and N.D. Mermin, Solid State Physics, Saunders College (Philadelphia) 1976.

[11] L.C. Biedenharn and J.D. Louck, Angular Momentum in Quantum Physics; Theory and Applications, Addison-Wesley (Reading) 1981.

[12] D.M. Brink and G.R. Satchler, Angular Momentum, Clarendon Press (Oxford) 1993.

[13] E.U. Condon and G.H. Shortley, The Theory of Atomic Spectra, University Press (Cambridge) 1951.

[14] A. Bohr and B.R. Mottelson, Nuclear Structure, W.A. Benjamin (New York) 1969.

[15] A. de-Shalit and H. Feshbach, Theoretical Nuclear Physics, Wiley (New York) 1974.

[16] C.J. Morningstar and M. Weinstein, Phys. Rev. Lett. 73, 1873 (1994); preprint SLACPUB-7121 (T) and hep-lat/9603016.

[17] M. Rotenberg, R. Bivins, N. Metropolis, and J.K. Wooten, The $3-j$ and $6-j$ Symbols, Technology Press (Cambridge, Mass.) 1959. 


\section{FIGURES}

FIG. 1. Distribution of strength for the ground state of the $2 \times 4$ Hamiltonian in the $S_{z}$ basis and in the plaquette basis.

FIG. 2. Distribution of strength for the first-excited state of the $2 \times 4$ Hamiltonian in the $S_{z}$ basis and in the plaquette basis. 


\section{TABLES}

TABLE I. Eigenvalues and eigenvectors of the $2 \times 2$ system.

\begin{tabular}{lccrc}
\hline \hline$l_{1}$ & $j_{1}$ & $\mathrm{E}$ & degeneracy \\
\hline 1 & 1 & 0 & -2 & 1 \\
1 & 1 & 1 & -1 & 3 \\
0 & 0 & 0 & 0 & 1 \\
0 & 1 & 1 & 0 & 3 \\
1 & 0 & 1 & 0 & 3 \\
1 & 1 & 2 & +1 & 5 \\
\hline \hline
\end{tabular}

TABLE II. Low-lying spectrum for the $2 \times 6$ Heisenberg ladder. The different approximations are explained in the text. Quantities in parenthesis represent the ratio to the exact value.

\begin{tabular}{llccr}
\hline \hline$S$ & Weak Coupling & Truncated & CORE (Range-2) & Exact \\
\hline 0 & $-6.000(0.909)$ & $-6.335(0.959)$ & $-6.588(0.998)$ & -6.603 \\
& $-4.000(0.818)$ & $-4.396(0.899)$ & $-4.899(1.002)$ & -4.888 \\
& $-4.000(0.836)$ & $-4.250(0.889)$ & $-4.882(1.021)$ & -4.783 \\
\hline 1 & $-5.000(0.844)$ & $-5.580(0.942)$ & $-5.928(1.001)$ & -5.924 \\
& $-5.000(0.909)$ & $-5.112(0.930)$ & $-5.458(0.993)$ & -5.498 \\
& $-5.000(0.983)$ & $-4.666(0.917)$ & $-5.132(1.009)$ & -5.087 \\
& $-4.000(0.797)$ & $-4.538(0.905)$ & $-5.092(1.015)$ & -5.017 \\
& $-4.000(0.850)$ & $-4.125(0.877)$ & $-4.809(1.022)$ & -4.705 \\
\hline 2 & $-4.000(0.823)$ & $-4.413(0.908)$ & $-4.862(1.000)$ & -4.862 \\
& $-4.000(0.925)$ & $-3.875(0.890)$ & $-4.426(1.017)$ & -4.352 \\
\hline 3 & $-3.000(0.895)$ & $-2.750(0.821)$ & $-3.414(1.019)$ & -3.351 \\
\hline \hline
\end{tabular}




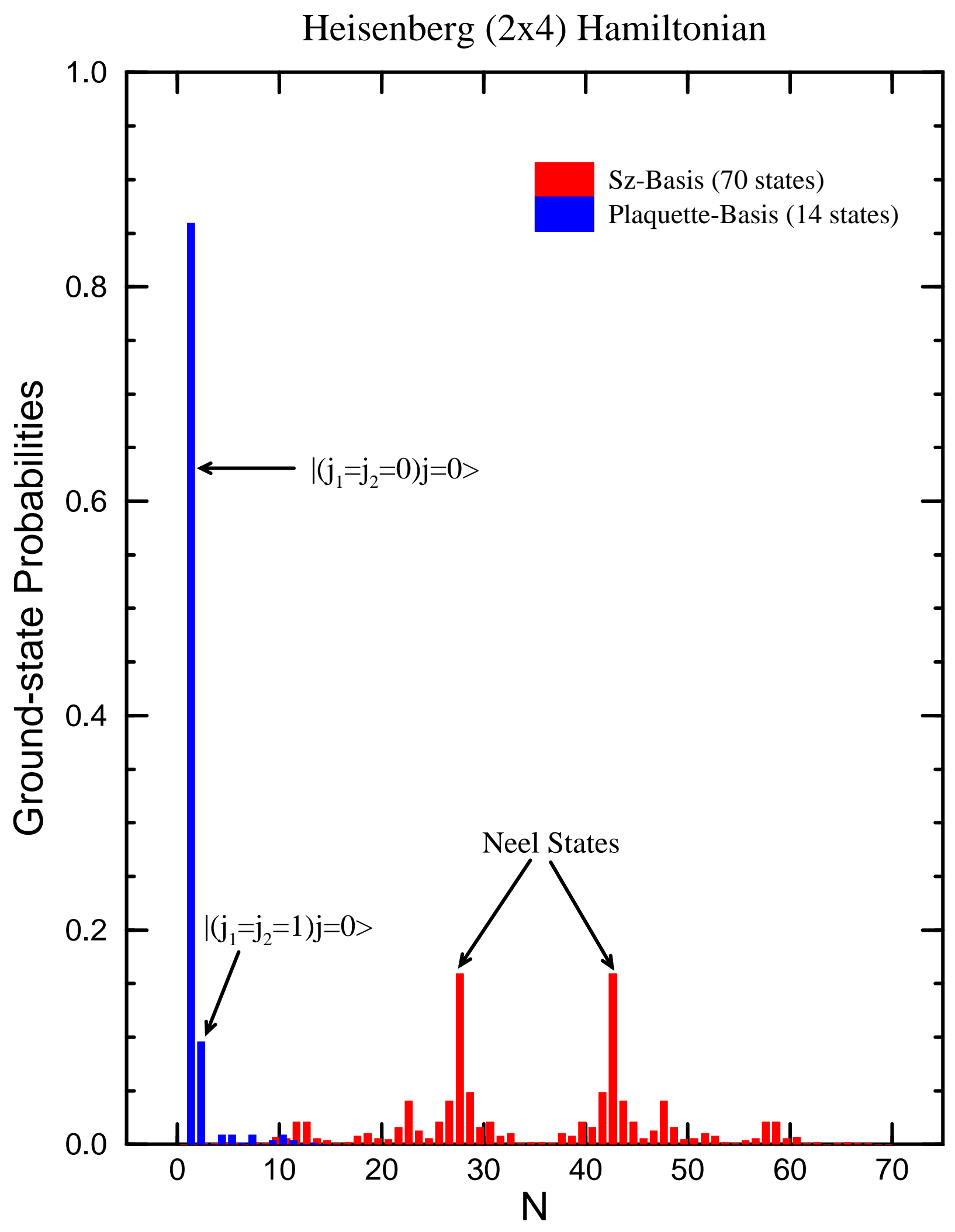




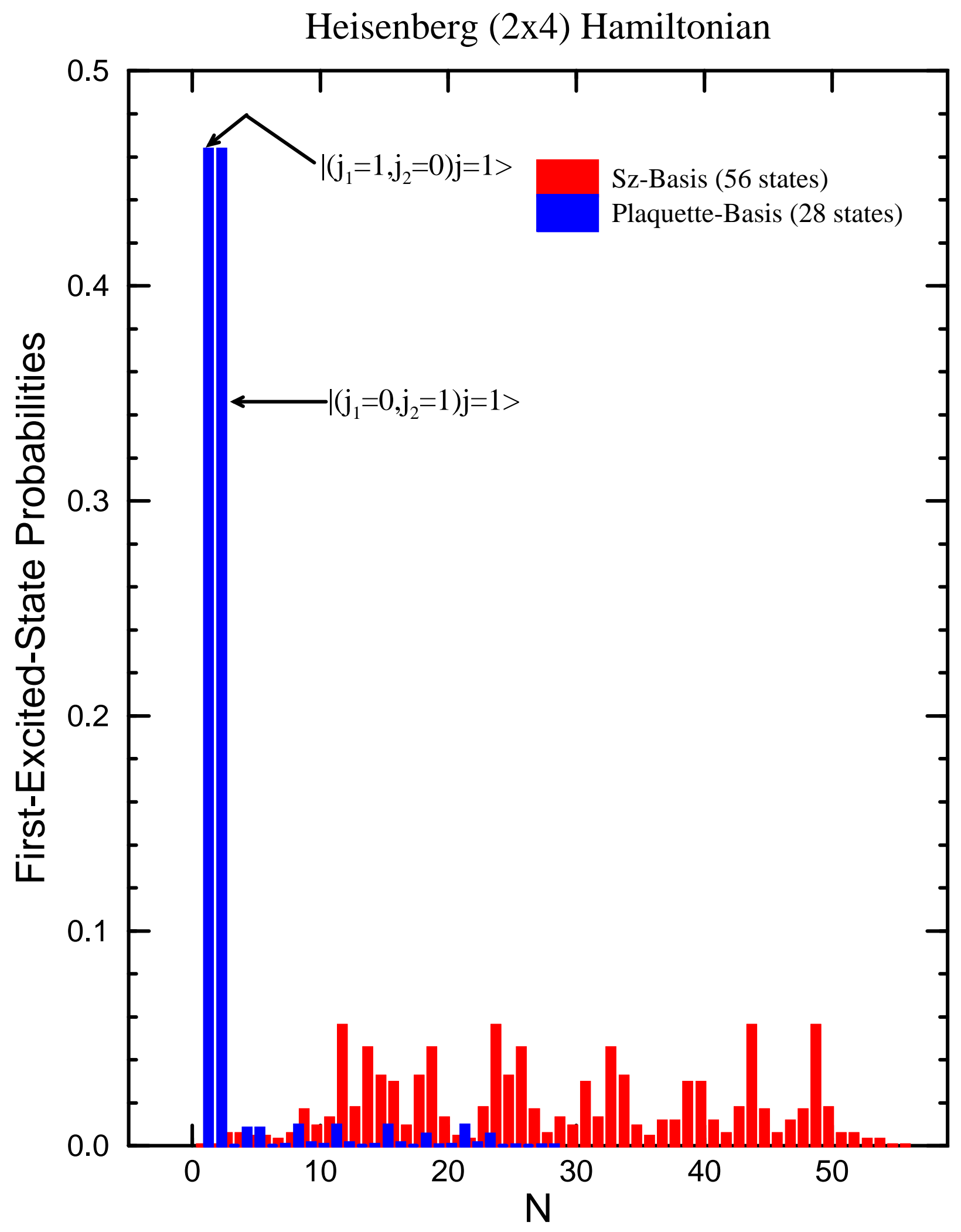

